\title{
Emergence of Magnetism in Doped Two-Dimensional Honeycomb Structures of III-V Binary Compounds
}

\author{
Krzysztof Zberecki
}

Received: 10 April 2012 / Accepted: 12 June 2012 / Published online: 27 June 2012

(C) The Author(s) 2012. This article is published with open access at Springerlink.com

\begin{abstract}
Using first-principles plane-wave calculations, a systematic study of the magnetic properties of doped twodimensional honeycomb structures of III-V binary compounds has been conducted, both for magnetic or nonmagnetic dopants. Calculations show that all cases where the magnetic moment is non-zero are energetically more favorable. For such cases band structure and (partial) density of states were calculated and analyzed in detail. The possible applications of these structures were also discussed.
\end{abstract}

Keywords Ab-initio $\cdot$ Two-dimensional $\cdot$ Magnetism

\section{Introduction}

Since its discovery in 2004 graphene [1] draws much attention because of unique features of this two-dimensional system. Graphene is composed of a $\mathrm{sp}^{2}$-bonded carbon atoms forming a honeycomb structure. It has a very interesting electronic structure with characteristic, linear energy dispersion near K point of the Brillouin zone. A summary of the subject can be found for example in [3].

Shortly after, experimental techniques allowed fabrication of other new two-dimensional materials, like BN and $\mathrm{MoS}_{2}$ honeycomb structures [2] or $\mathrm{ZnO}$ monolayers [8]. The discovery of such stable two-dimensional material like graphene triggered search for similar structures made from different compounds. Up to now many of these hypothetical structures constructed from silanene (2D Si) and germanene

K. Zberecki $(\bowtie)$

Faculty of Physics, Warsaw University of Technology,

ul. Koszykowa 75, 00-662 Warsaw, Poland

e-mail: zberecki@if.pw.edu.pl
(2D Ge) [4, 5], III-V compounds [6], $\mathrm{SiC}$ [7] or $\mathrm{ZnO}$ [9] have been studied theoretically.

On the other hand graphene and other nano-scale materials are recognized as future building blocks of new electronics technologies [10], including spintronics (e.g. [11]). In the case of low (one- and two-) dimensional structures problem arises because of famous Mermin-Wagner theorem [12], which prevents ferro- or antiferromagnetic order to occur in finite temperatures, which is essential for spintronics and other modern applications. This started the theoretical and experimental search for magnetism in graphene and other structures. One of the most promising directions is the emergence of magnetism in such structures as an effect of presence of local defects [13]. According to work of Palacios et al. [14] and, independently, of Yazyev [15, 16] single-atom defects can induce ferromagnetism in graphene-based materials. In both cases, the magnetic order arises as an effect of the presence of single-atom defects in combination with a sublattice discriminating mechanism, in agreement with Lieb's theorem [17, 18]. Based on these findings several theoretical studies have been conducted in search for magnetism in low-dimensional structures either for graphene and BN [19] or other (hypothetical) structures like SiC [7].

In this paper the influence of local defects on magnetic structure of two-dimensional honeycomb structures of $\mathrm{GaN}$, AlN and InN has been analyzed by means of ab-initio calculations. Since bulk versions of these compounds are very important semiconductors in today's electronics it would be interesting to check whether such two-dimensional materials could have non-zero magnetic moment. Despite of the fact that neither of them have been yet synthesized, calculated cohesion energies [6] suggest that such structures would be stable and their experimental procurement is highly probable.

Next section contains computational details followed by results. Last section concludes this work. 


\section{Computational Details}

To investigate magnetic properties of $\mathrm{GaN}, \mathrm{AlN}$ and $\mathrm{InN}$ honeycomb structures a series of ab-initio calculations have been conducted with use of DFT VASP code [20, 21] with PAW potentials [22]. For both spin-unpolarized and spin-polarized cases the exchange-correlation potential has been approximated by generalized gradient approximation (GGA) using PW91 functional [23]. Kinetic energy cutoff of $500 \mathrm{eV}$ for plane-wave basis set has been used. Supercells of size $3 \times 3 \times 1$ have been checked to be large enough to prevent defects to interact with its periodic image. ${ }^{1}$ In all cases for self-consistent structure optimizations, the Brillouin zone (BZ) was sampled by $20 \times 20 \times 1$ special $k$ points. All structures have been optimized for both, spin-unpolarized and spin-polarized cases unless FeynmanHellman forces acting on each atom become smaller than $10^{-4} \mathrm{eV} / \AA$. A vacuum spacing of $12 \AA$ was applied to hinder the interactions between monolayers in adjacent supercells. Calculated lattice constants are in agreement with [6].

\section{Results}

As mentioned, non-magnetic honeycomb sheets can attain spin-polarized states due to the presence of local defects. In this work two kinds of defects have been analyzedvacancies and substitutions.

For all three compounds a vacancy was generated first by removing a single atom, $\mathrm{Al}, \mathrm{Ga}$, In or $\mathrm{N}$ from each supercell, then the atomic structure was optimized. In all cases structures with single $\mathrm{N}$ vacancy are non-magnetic, while $\mathrm{Al}, \mathrm{Ga}$ or In vacancies induce non-zero magnetic moment, equal to $3.00 \mu_{\mathrm{B}}$.

Bonding in typical 2D honeycomb structure consists of $\sigma$ and $\pi$ states, the former are $\mathrm{sp}^{2}$ hybrids while the latter are $\mathrm{p}_{z}$ orbitals. This strong $\pi$ bonding between two nearest neighbor $\mathrm{p}_{z}$ orbitals makes the structure planar [6]. Covalent $\sigma$ bonds between adjacent atoms are in-plane.

In the case of AlN (unlike in graphene, [15]) Al vacancy does not break the local threefold symmetry described above. The three adjacent $\mathrm{N}$ atoms are left with one dangling bond each, unsaturated, each providing a contribution of magnitude $1 \mu_{\mathrm{B}}$ to the magnetic moment. In the case of $\mathrm{N}$ vacant structures magnetic moment is equal to 0 due to the fact that all electrons in adjacent $\mathrm{Al}$ atoms are used to make bond with other $\mathrm{N}$ atoms, so there is no unpaired electrons left. Figure 1 shows the density of states (DoS) for spin-polarized Al-vacant AlN, on which difference between

\footnotetext{
${ }^{1}$ For chosen structures, calculations within supercell $5 \times 5 \times 1$ were conducted, giving the same value of magnetic moment and similar density of states.
}

DoS AlN-Al 2D

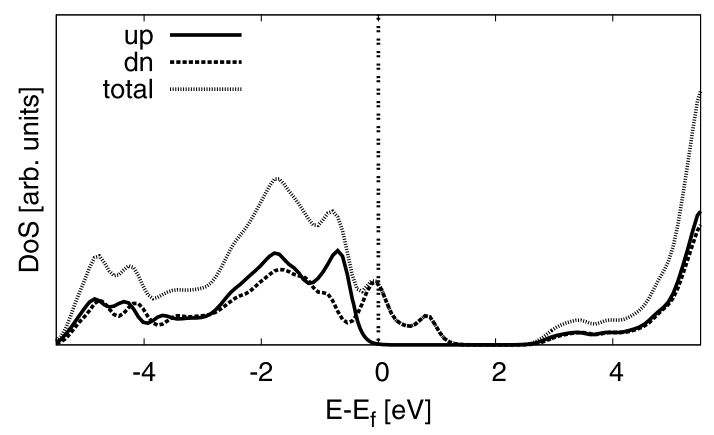

Fig. 1 Density of states of Al-vacant AlN

majority spin (up) and minority spin (dn) in the vicinity of Fermi level (horizontal line) can be observed which is the main source of non-zero magnetic moment. Analysis of the calculated partial magnetization shows that all magnetic moment is situated on p-states of $\mathrm{N}$ atoms located in the area of vacancy.

In the case of substitution the procedure was as follows. For all three compounds various single foreign atoms have been substituted, then structure has been optimized. In the case of $\mathrm{AlN}(\mathrm{GaN}), \mathrm{Al}(\mathrm{Ga})$ atom has been substituted by atoms from fourth period of periodic table from $\mathrm{K}$ to $\mathrm{Zn}$ (including $\mathrm{Na}$ and $\mathrm{Mg}$ for $\mathrm{AlN}$ ). In the case of $\mathrm{InN}$, In has been substituted by atoms from fifth period of periodic table from $\mathrm{Rb}$ to $\mathrm{Cd}$ (excluding $\mathrm{Tc}$ ). In all three compounds, $\mathrm{N}$ has been substituted by $\mathrm{C}, \mathrm{B}$ and $\mathrm{P}$ atoms. Table 1 shows calculated magnetic moments and differences between total energy of spin-unpolarized and spin-polarized states, $\Delta E=E_{n s p}-E_{s p}$, i.e. positive value of $\Delta E$ means that spin-polarized state is more energetically favored. This is the case for all compounds with non-zero magnetic momentthe largest energy differences (up to $1.25 \mathrm{eV}$ ) are for instances with highest values of magnetic moment. For AIN highest values of induced magnetic moment are in case of Mn, Co $\left(4.00 \mu_{\mathrm{B}}\right)$ and for Fe $\left(4.26 \mu_{\mathrm{B}}\right)$. Similar situation can be observed for GaN doped with $\mathrm{Mn}$, Co and Fe. With decreasing number of d-shell electrons the value of magnetic moment drops as well as for the case of $\mathrm{Zn}$ which has d-shell closed. In the case of InN this tendency holds although values of magnetic moments are much smaller, being the highest for Ru and Rh. Figures 3 and 4 show mechanism of generation of magnetic moment in the case of GaN doped with Ni. Left plot of Fig. 3 shows the band structure of Ni-doped GaN vs. undoped one (which is a semiconductor with bandgap equal to $2.30 \mathrm{eV}$, calculated within GGA) in spin-unpolarized case. Formation of doping bands in the vicinity of Fermi level can be observed. The top right plot shows band structure of $\mathrm{GaN}+\mathrm{Ni}$ in the spin-polarized case, where a large splitting of these bands between spin up and down bands can be observed. Top plots of Fig. 4 show 
density of states for spin polarized case. Right one shows total DoS vs. DoS of spin up and down. Fermi energy is almost exactly in the middle of splitter up and down DoS. Left plot shows the vicinity of Fermi energy more closely, where large splitting of up and down DoS can be observed. Since almost all electrons occupying vicinity of the Fermi level are d-shell electrons, which can be read from partial density of states (bottom left plot) the mechanism of magnetic moment emergence becomes clear-all magnetic moments reside on unpaired d-electrons of dopant atom. Calculations show that this mechanism is universal for all structures having non-zero magnetic moment doped with transition metal elements.

In the case of doping with alkali metal elements and alkaline earth metal elements only in Na- and K-doped AlN calculations show non-zero magnetic moment (1.88 and $1.70 \mu_{\mathrm{B}}$, respectively, for $\mathrm{Na}$ and $\mathrm{K}$ ). The mechanism of formation of magnetic moment is similar to the case of vacant structures (since $\mathrm{Na}$ and $\mathrm{K}$ have only one valence elec-

$\mathrm{GaN}+\mathrm{Ni} 2 \mathrm{D}$ sp

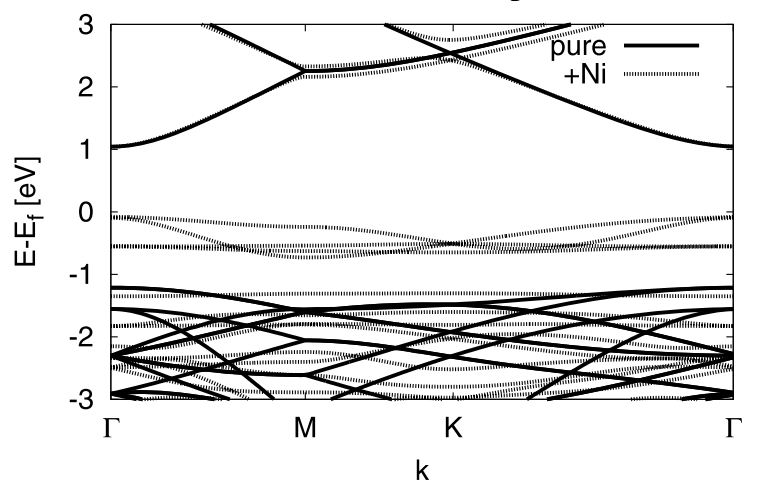

tron). Figure 2 shows DoS for spin-polarized Na-doped AlN, on which difference between spin up and spin down in the vicinity of Fermi level can be observed.

This is similar to the situation depicted on Fig. 1 although in the case of Na-doped structure splitting is smaller.

DoS AlN+Na 2D

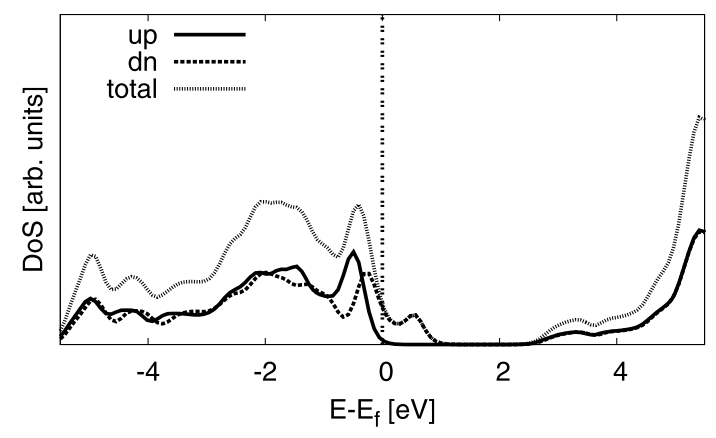

Fig. 2 Density of states of Na-doped AlN

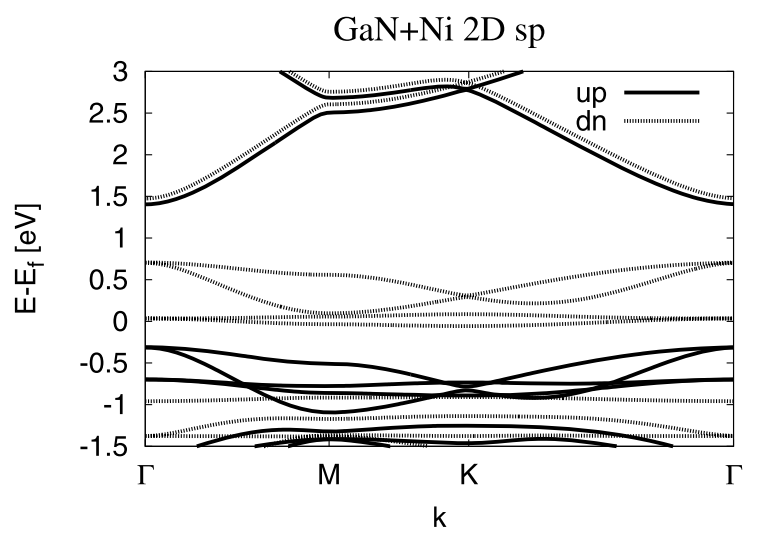

Fig. 3 Band structure of Ni-doped GaN. Details in text

Table 1 Magnetic moments and total energy differences between spin up and spin down states for different transition metal elements dopants

\begin{tabular}{|c|c|c|c|c|c|c|c|c|c|c|c|c|c|c|}
\hline \multicolumn{15}{|l|}{ AlN } \\
\hline for $\mathrm{Al}$ & $\mathrm{Na}$ & $\mathrm{Mg}$ & $\mathrm{K}$ & $\mathrm{Ca}$ & $\mathrm{Sc}$ & & V & $\mathrm{Cr}$ & $\mathrm{Mn}$ & $\mathrm{Fe}$ & Co & $\mathrm{Ni}$ & $\mathrm{Cu}$ & $\mathrm{Zn}$ \\
\hline$\mu\left(\mu_{\mathrm{B}}\right)$ & 1.88 & 0.00 & 1.70 & 0.00 & 0.00 & & 0.00 & 2.98 & 3.99 & 4.26 & 3.96 & 2.98 & 1.85 & 0.00 \\
\hline$\Delta E(\mathrm{eV})$ & 0.06 & 0.00 & 0.04 & 0.00 & 0.00 & & 0.00 & 0.70 & 0.98 & 1.09 & 0.94 & 0.38 & 0.06 & 0.00 \\
\hline \multicolumn{15}{|l|}{$\mathrm{GaN}$} \\
\hline for $\mathrm{Ga}$ & & $\mathrm{K}$ & $\mathrm{Ca}$ & Sc & & $\mathrm{Ti}$ & $\mathrm{V}$ & $\mathrm{Cr}$ & $\mathrm{Mn}$ & $\mathrm{Fe}$ & Co & $\mathrm{Ni}$ & $\mathrm{Cu}$ & $\mathrm{Zn}$ \\
\hline$\mu\left(\mu_{\mathrm{B}}\right)$ & & 0.00 & 0.00 & 0.00 & & 0.90 & 1.72 & 2.99 & 3.99 & 4.51 & 3.97 & 2.97 & 1.32 & 0.00 \\
\hline$\Delta E(\mathrm{eV})$ & & 0.00 & 0.00 & 0.00 & & 0.12 & 0.07 & 0.66 & 1.15 & 1.25 & 0.91 & 0.30 & 0.01 & 0.00 \\
\hline \multicolumn{15}{|l|}{ InN } \\
\hline for In & & $\mathrm{Rb}$ & $\mathrm{Sr}$ & $\mathrm{Y}$ & & $\mathrm{Zr}$ & $\mathrm{Nb}$ & Mo & $\mathrm{Tc}$ & $\mathrm{Ru}$ & $\mathrm{Rh}$ & $\mathrm{Pd}$ & $\mathrm{Ag}$ & $\mathrm{Cd}$ \\
\hline$\mu\left(\mu_{\mathrm{B}}\right)$ & & 0.00 & 0.00 & 0.00 & & 0.00 & 0.99 & 0.39 & - & 1.38 & 1.91 & 0.42 & 0.00 & 0.00 \\
\hline$\Delta E(\mathrm{eV})$ & & 0.00 & 0.00 & 0.00 & & 0.00 & 0.16 & 0.01 & - & 0.04 & 0.10 & 0.01 & 0.00 & 0.00 \\
\hline
\end{tabular}



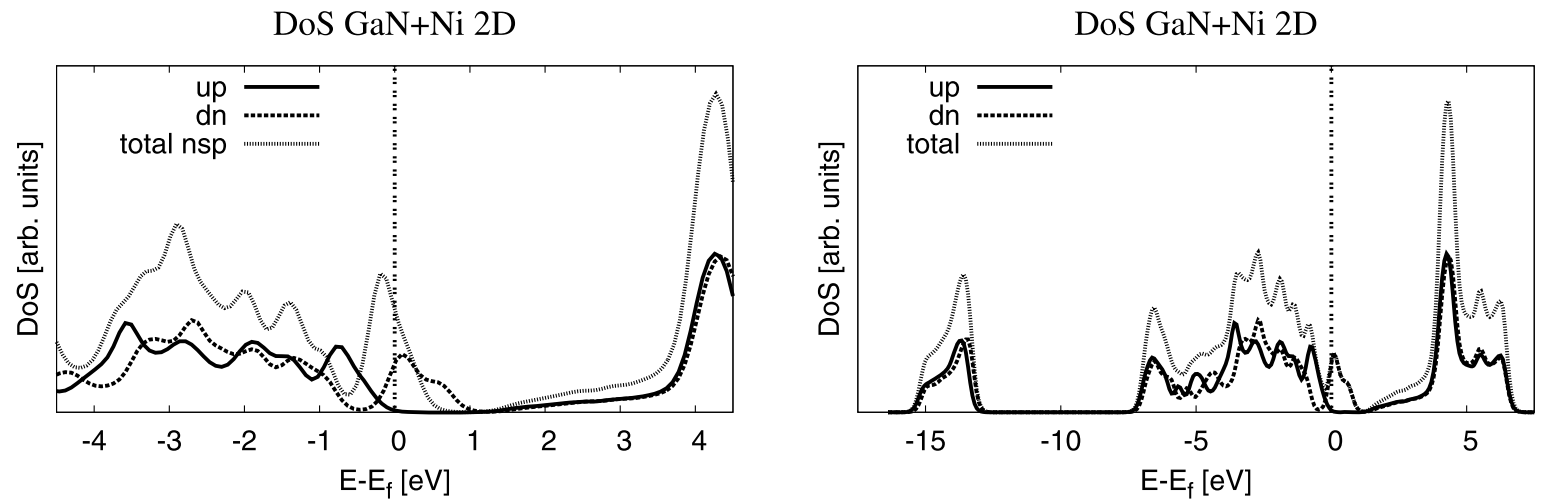

\section{DoS GaN+Ni 2D}

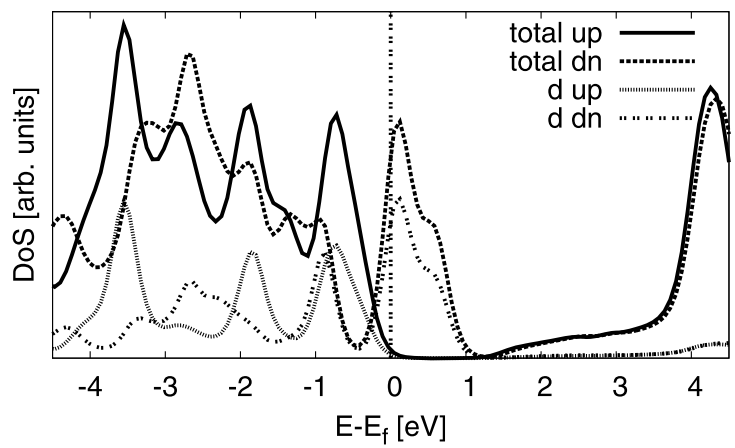

Fig. 4 Density of states for Ni-doped GaN. Details in text

Table 2 Magnetic moments and total energy differences between spin up and spin down states for different dopants

\begin{tabular}{llll}
\hline AlN & & & \\
\hline for N & $\mathrm{B}$ & $\mathrm{C}$ & $\mathrm{P}$ \\
$\mu\left(\mu_{\mathrm{B}}\right)$ & 0.00 & 1.00 & 0.00 \\
$\Delta E(\mathrm{eV})$ & 0.00 & 0.14 & 0.00 \\
\hline $\mathrm{GaN}$ & & & \\
\hline for $\mathrm{N}$ & $\mathrm{B}$ & $\mathrm{C}$ & $\mathrm{P}$ \\
$\mu\left(\mu_{\mathrm{B}}\right)$ & 0.00 & 1.00 & 0.00 \\
$\Delta E(\mathrm{eV})$ & 0.00 & 0.11 & 0.00 \\
\hline $\mathrm{InN}$ & & & \\
\hline for N & $\mathrm{B}$ & $\mathrm{C}$ & $\mathrm{P}$ \\
$\mu\left(\mu_{\mathrm{B}}\right)$ & 0.00 & 1.00 & 0.00 \\
$\Delta E(\mathrm{eV})$ & 0.00 & 0.10 & 0.00 \\
\hline
\end{tabular}

In case of substitution of $\mathrm{N}$ atom by $\mathrm{C}, \mathrm{B}$ and $\mathrm{P}$, only $\mathrm{C}$ doped structures had non-zero magnetic moment, which was equal to $1.00 \mu_{\mathrm{B}}$ in all compounds. As can be seen from Table 2, magnetic states in all cases are lower by about $0.1 \mathrm{eV}$ than non-magnetic states.

\section{Conclusions}

Ab-initio calculations have been conducted for vacancy and substitution defects in honeycomb AlN, GaN and InN compounds. Calculations show that in all three compounds vacancy of $\mathrm{Al}, \mathrm{Ga}$ or In, respectively, gives magnetic moment of $3.00 \mu_{\mathrm{B}}$, which is an interesting conclusion from application point of view. On the other hand substitution of $\mathrm{Al}$ or $\mathrm{Ga}$ by transition metal elements ( $\mathrm{Mn}, \mathrm{Fe}, \mathrm{Co}$ ) can give an even higher value of magnetic moment $\left(4.00 \mu_{\mathrm{B}}\right)$. Since technique of implantation of metal atoms into 2D surface has been recently reported [24], it is also very promising direction. On the other hand substitution by non-metallic atoms or substitution of nitrogen atoms by IV or V group atoms does not give a significant magnetic moment. These results may give a hint for experimentalists searching two-dimensional magnetic materials.

Acknowledgements Numerical calculations were performed at the Interdisciplinary Centre for Mathematical and Computational Modeling (ICM) at Warsaw University.

Open Access This article is distributed under the terms of the Creative Commons Attribution License which permits any use, distribution, and reproduction in any medium, provided the original author(s) and the source are credited. 


\section{References}

1. Novoselov, K.S., et al.: Science 306, 666 (2004)

2. Novoselov, K.S., et al.: Proc. Natl. Acad. Sci. USA 102, 10451 (2005)

3. Castro Neto, A.H., et al.: Rev. Mod. Phys. 81, 109 (2009)

4. Lebegue, S., et al.: Phys. Rev. B 79, 115409 (2009)

5. Cahangirov, S., et al.: Phys. Rev. Lett. 102, 236804 (2009)

6. Sahin, H., et al.: Phys. Rev. B 80, 155453 (2009)

7. Bakaroglu, E., et al.: Phys. Rev. B 81, 075433 (2010)

8. Tusche, C., et al.: Phys. Rev. Lett. 99, 026102 (2007)

9. Topsakal, M., et al.: Phys. Rev. B 80, 235119 (2009)

10. Avouris, P., et al.: Nat. Nanotechnol. 2, 605 (2007)

11. Zutic, I., et al.: Rev. Mod. Phys. 76, 323 (2004)
12. Mermin, N.D., Wagner, H.: Phys. Rev. Lett. 17, 1133 (1966)

13. Esquinazi, P.: Phys. Rev. Lett. 91, 227201 (2003)

14. Palacios, J.J., et al.: Phys. Rev. B 77, 195428 (2008)

15. Yazyev, O.: Phys. Rev. Lett. 101, 037203 (2008)

16. Yazyev, O., et al.: Phys. Rev. B 75, 125408 (2007)

17. Lieb, E.H.: Phys. Rev. Lett. 62, 1201 (1989)

18. Lieb, E.H.: Phys. Rev. Lett. 62, 1927(E) (1989)

19. Ataca, C., et al.: Phys. Rev. B 82, 165402 (2010)

20. Kresse, G., Hafner, J.: Phys. Rev. B 47, 558 (1993)

21. Kresse, G., Furthmuller, J.: Phys. Rev. B 54, 11169 (1996)

22. Blochl, E.: Phys. Rev. B 50, 17953 (1994)

23. Perdew, J.P., et al.: Phys. Rev. B 46, 6671 (1992)

24. Lahiri, J., et al.: Nat. Nanotechnol. 5, 326 (2010) 